\title{
Preparation of 3D Architecture Graphdiyne Nanosheets for High- Performance Sodium-Ion Batteries and Capacitors
}

\author{
Kun Wang, ${ }^{\dagger, \S}$ Ning Wang, ${ }^{\dagger, \S}$ Jianjiang He, ${ }^{\dagger}$ Ze Yang, ${ }^{\dagger}$ Xiangyan Shen, ${ }^{\dagger,}$ and Changshui Huang, ${ }^{\dagger}{ }^{\dagger}$ \\ ${ }^{\dagger}$ Qingdao Institute of Bioenergy and Bioprocess Technology, Chinese Academy of Sciences, Qingdao 266101, China \\ "University of Chinese Academy of Sciences, No. 19A Yuquan Road, 100049 Beijing, China
}

Supporting Information

ABSTRACT: Here, we apply three-dimensional (3D) architecture graphdiyne nanosheet (GDY-NS) as anode materials for sodium-ion storage devices achieving high energy and power performance along with excellent cyclic ability. The contribution of $3 \mathrm{D}$ architecture nanostructure and intramolecular pores of the GDY-NS can substantially optimize the sodium storage behavior through the accommodated intramolecular pore, 3D interconnective porous structure, and increased activity sites to facilitate a fast sodium-iondiffusion channel. The contribution of butadiyne linkages and the formation of a stable solid electrolyte interface layer are directly confirmed through the in situ Raman measurement. The GDY-NSbased sodium-ion batteries exhibit a stable reversible capacity of approximately $812 \mathrm{mAh} \mathrm{g}^{-1}$ at a current density of $0.05 \mathrm{~A} \mathrm{~g}^{-1}$; they

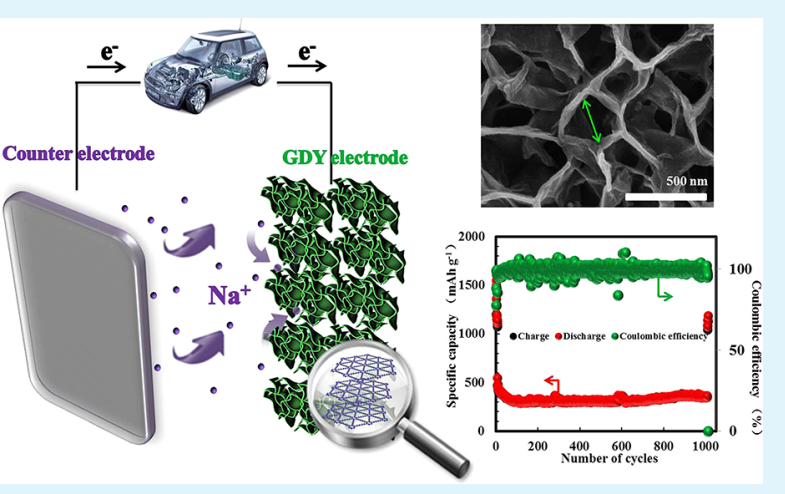
maintain more than $405 \mathrm{mAh} \mathrm{g}^{-1}$ over 1000 cycles at a current density of $1 \mathrm{~A} \mathrm{~g}^{-1}$. Furthermore, the sodium-ion capacitors could deliver a capacitance more than $200 \mathrm{~F} \mathrm{~g}^{-1}$ over 3000 cycles at $1 \mathrm{~A} \mathrm{~g}^{-1}$ and display an initial specific energy as high as $182.3 \mathrm{Wh}$ $\mathrm{kg}^{-1}$ at a power density of $300 \mathrm{~W} \mathrm{~kg}^{-1}$ and maintain specific energy of $166 \mathrm{Wh} \mathrm{kg}^{-1}$ even at a power density of $15000 \mathrm{~W} \mathrm{~kg}^{-1}$. The high energy and power density along with excellent cyclic performance based on the GDY-NS anode offers a great potential toward application on next-generation energy storage devices.

KEYWORDS: graphdiyne, 3D architecture, sodium-ion, batteries, capacitors, high-performance

\section{INTRODUCTION}

With continuously increasing demand for battery performance, lithium-ion batteries (LIBs) cannot completely satisfy the rapid growth requirement for high energy density, high power density, and low cost, owing to the limits of the existing material system and restricted lithium resources. ${ }^{1-3}$ Thus, it is significant and urgent to find a substitute battery system to meet the growing demands. Sodium, located adjacent to the lithium in the periodic table of the elements, is considered as one of the most attractive elements for replacing lithium for its abundant reserves in the earth crust (4-5 times more than lithium) and similar chemical properties. ${ }^{4,5}$ These advantages make great potential for sodium energy storage devices as most promising candidates to replace LIBs for application of renewable energy storage and smart grids. In recent years, many efforts have been made to enrich and develop the sodium energy storage materials. ${ }^{6}$ Layered transition-metal oxides, ${ }^{7}$ phosphates with $\mathrm{Fe}(\mathrm{II})$ and $\mathrm{Mn}(\mathrm{II}){ }^{8,9}$ or organic compounds ${ }^{10}$ have been widely studied for reversibly storage of sodium. For anode materials, the carbonaceous materials, accompanied with the advantage of abundant resource, stability, and low cost have been mostly investigated. Because of the larger ionic radius of $\mathrm{Na}^{+}(102 \mathrm{pm})$ than that of $\mathrm{Li}^{+}(76 \mathrm{pm})$ and the lower kinetics of reversible sodiation/desodiation compared to that of $\mathrm{Li}^{+}$, it is necessary to choose suitable carbonaceous materials with an appropriate structure to achieve a large electrode-electrolyte contact interface, a short ion-diffusion pathway, an enhanced reaction activity, and better accommodation of the insertion and extraction-induced strain for sodium ions. ${ }^{11-13}$ Carbonaceous nanomaterials, such as hard carbon nanofiber, ${ }^{14}$ hollow carbon nanospheres, ${ }^{15}$ nanoparticles, ${ }^{16}$ and graphene, ${ }^{17,18}$ were widely studied, achieving a moderate capacity between 100 and $300 \mathrm{mAh} \mathrm{g}^{-1}$ of sodium-ion batteries (NIBs). ${ }^{19}$ However, further improving the performance of sodium active sites and interlayer spacing are still major issues for these materials to be applied in high-performance sodium storage devices. Meanwhile, application of hybrid capacitors is another approach for achieving the high rate performance and cycling capability. ${ }^{20}$ Replacement of lithium with sodium to construct the sodiumion capacitors (NICs) also offers the opportunity to build the device integrated with the features of potentially low cost and high electrochemical performance. ${ }^{21}$ To combine the advantages of both batteries and capacitors, sodium-based electrochemical capacitors (NICs) have been proposed and act as a fascinating alternative for future energy storage for improving

Received: August 1, 2017

Accepted: October 25, 2017

Published: October 25, 2017 
the capacity, poor rate, and cycle capability of sodium-based energy storage devices. ${ }^{22,23}$

Recently, graphdiyne (GDY) as a newly discovered carbon allotrope, has attracted considerable attention for its promising electronic, optical, and mechanical properties. ${ }^{24-27}$ The presence of the $\mathrm{sp}$ - and $\mathrm{sp}^{2}$-hybridized carbon atoms and butadiyne linkages in GDY disrupts the regular hexagonal crystal lattice of the traditional graphene layer. ${ }^{28}$ Therefore, the GDY materials are considered to show a layered structure with intramolecular pores, which are expected to greatly increase active bonding sites and facilitate fast ion-diffusion channels for sodium-ion storage compared with other carbon-based materials, like graphene or expanded graphite, impelling their electrochemical performance to high capacities and excellent cycling capability. ${ }^{29-31}$ Density functional theory and firstprinciples calculations have already indicated the maximum sodium concentration is far exceeding the upper limit of sodium insertion into graphite (similar to $\mathrm{NaC}_{6}$ as normal carbon material). ${ }^{32}$ Although bulk GDY has been applied for the NIB anode, the result was lower than the theoretical prediction, probably due to the insufficient quality and stack of layers of the GDY. ${ }^{33}$ Nevertheless, the recent report about the assembled GDY nanosheets (GDY-NSs) through a modified Glaser-Hay coupling reaction motivates us to obtain a threedimensional (3D) structured GDY composed with a hierarchical porous and continuous vertical network. ${ }^{34}$ GDYNS is expected to interconnect innumerable active sites and promote the fast transfer of ions between GDY layered frameworks. It is reasonable to consider that the unique 3D architecture of GDY is able to improve the rapid transportation, migration, and diffusion, of sodium in the phase from a GDY molecular monolayer to a 3D architecture GDY bulk network. Therefore, in this work, we demonstrate the first successful application of GDY-NS as an anode material in practical NIBs and NICs to exhibit the remarkable sodium storage property. The results confirm that the as-prepared GDY-NS-based NIBs show a high reversible capacity of approximately $812 \mathrm{mAh} \mathrm{g}^{-1}$ at a current density of $0.05 \mathrm{~A} \mathrm{~g}^{-1}$ and even $405 \mathrm{mAh} \mathrm{g}^{-1}$ over 1000 cycles at a current density of $1 \mathrm{~A} \mathrm{~g}^{-1}$. Besides, GDY-NS based NICs deliver a high capacitance of more than $200 \mathrm{~F} \mathrm{~g}^{-1}$ over 3000 cycles at $1 \mathrm{~A} \mathrm{~g}^{-1}$, with active carbon as the cathode. Meanwhile, the NICs also exhibit an initial specific energy as high as $182.3 \mathrm{Wh} \mathrm{kg}^{-1}$ at a power density of 300 and $166 \mathrm{Wh}$ $\mathrm{kg}^{-1}$ at a power density of $15000 \mathrm{~W} \mathrm{~kg}^{-1}$, presenting the benefit of better sodium storage and delivery ability for GDYNS. The relationship between the excellent electrochemical performance and the 3D architecture and multiporous structure of GDY-NS is sufficiently researched. All of the results can enrich the research of the energy storage materials.

\section{EXPERIMENTAL SECTION}

Preparation of GDY-NS. GDY-NS was obtained following the optimized synthetic route via a modified Glaser-Hay coupling reaction, as reported. ${ }^{34}$ The precursor hexakis(trimethylsilyl)ethynyl benzene (HEB-TMS) was obtained following the reported synthetic route. ${ }^{28}$ The HEB monomer could be obtained after deprotection of HEB-TMS by tetrabutylammonium fluoride and used immediately to isolate moisture and air. The copper foil was carefully dipped in $0.1 \mathrm{M}$ hydrochloric acid overnight and washed with distilled water, ethanol, and acetone several times, then dried under argon. Several (more than 10) pieces of the copper foil were cut into a $3 \times 3 \mathrm{~cm}^{2}$ shape and added to the mixed solution of acetone, pyridine, and tetramethylethylenediamine, with an optimal volume ratio of 100:10:10 in a flask. Thirty five milligrams of HEB was dissolved with another $50 \mathrm{~mL}$ of acetone and added immediately into the mixed solution. Then, the mixture was stirred and kept under an argon atmosphere at $80{ }^{\circ} \mathrm{C}$ through a condenser for more than $12 \mathrm{~h}$. After the reaction, GDY-NS was grown on the surface of the copper foil. Finally, the copper plates were washed with heated acetone and ethanol several times and dried under nitrogen. The average mass loading of GDY-NS active materials at a range of $0.13-0.08 \mathrm{mg} \mathrm{cm}^{-2}$ (with different reaction conditions) was calculated by completely dissolving a certain area of the GDY-NScontained copper foil in a $6 \mathrm{M}$ hydrochloric acid solution with a small amount of ammonium persulfate to dissolve copper gradually; the exfoliated GDY-NS sheet was collected and washed carefully to remove the metal ions and impurities, then dried in vacuum. After collecting the GDY-NS sheet exfoliated from the $\mathrm{Cu}$ foil, the weight per unit area of the GDY-NS active materials was calculated.

Assembly of NIB Half-Cells and NIC Devices. The NIB halfcells assembled to measure the electrochemical performances of GDYNS and the sodium foil were used as the counter and reference electrode. One molar NaPF6 dissolved in 1:1 (v/v) mixture of ethylene carbonate and dimethyl carbonate was employed as the electrolyte in standard CR2032-type coin cells and used compressing sodium foils as counter electrodes separated by a glass fiber filters (GF/D, Whatman). The current densities and capacities were calculated on the basis of the GDY-NS active materials. The obtained GDY-NS was punched into $1 \mathrm{~cm}$ diameter as the binder-free electrodes both applied for NIBs and NICs with a calculated mass loading of $0.08 \mathrm{mg}$ for each electrode. Although the thin GDY-NS layer $(400 \mathrm{~nm})$ lead to a poor mass loading, it is helpful to study the thin and few-layer sodium intercalation in GDY-NS materials. For NICs, the obtained GDY-NS was firstly assembled as an NIB half-cell with a sodium foil and discharged to $0.005 \mathrm{~V}$ to obtain the presodiation GDY-NS. Here, the commercial activated carbon (AC, YP-50 Kuraray Co., Ltd., Japan), Super P, and binder (10\% poly(tetrafluoroethylene)) were mixed together at a weight ratio of $8: 1: 1$, rolled into thin sheets, then punched into $1 \mathrm{~cm}$ diameter. NICs were also assembled in CR2032-type cells with presodiation GDY-NS and extra sodium metal as negative materials and the AC positive electrode in the same electrolyte separated by a glass fiber filter. The mass ratio of cathode/anode was changed from 1:1 to 20:1 by adjusting the quality of the activated carbon electrode. ${ }^{34}$ In the case of NIC, the current densities and capacities were normalized by the total electroactive mass in both electrodes (GDY-NS and AC). Herein, the specific capacitance of NICs was calculated according to the following equation

$$
C=I t / 2 m \Delta V
$$

where $I$ is the discharge current (A), $t$ is the discharge time (s), $m$ is the total mass of active materials (GDY-NS and AC), and $\Delta V$ is the potential window $(\mathrm{V})$ of the discharge process. The Ragone plots of the assembled NIC were obtained by calculating the energy density and power density from the galvanostatic discharge curves according to the following equations

$$
\begin{aligned}
& P=V \times i \\
& E=P \times t / 3600 \\
& V=\left(V_{\max }+V_{\min }\right) / 2
\end{aligned}
$$

where $i$ is the current density normalized by the total electroactive mass in both electrodes $\left(\mathrm{A} \mathrm{g}^{-1}\right), t$ is the discharge time (s), and $V_{\max }$ and $V_{\min }$ are the potentials at the beginning and the end of the discharge $(\mathrm{V})$, respectively.

Materials Characterization. The GDY-NS films were transferred to a copper screen, and then the samples were characterized using field emission scanning electron microscopy (SEM, Hitachi S-4800) and transmission electron microscopy (TEM, H-7650) apparatus. The multimode atomic force microscope (AFM), Agilent 5400, with a silicon probe OSCM-PT- $\mathrm{R}_{3}$ (Bruker probes) was used to image the morphology of samples at room temperature in an ambient atmosphere. The X-ray diffraction (XRD) pattern was obtained by Bruker D8 ADVANCE diffractometer with $\mathrm{Cu} \mathrm{K} \alpha$ irradiation at $\lambda=$ 

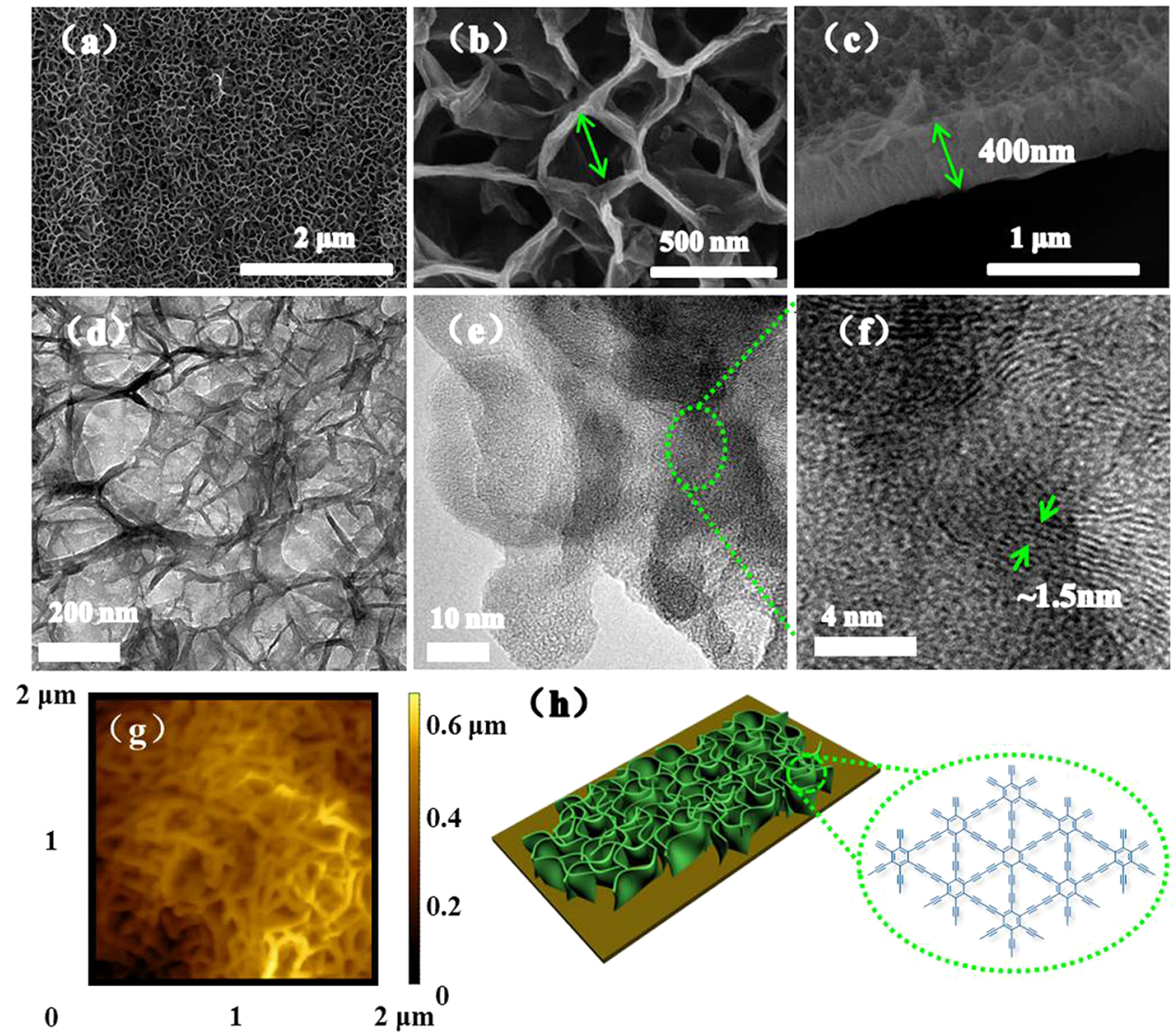

Figure 1. Morphologies of GDY-NS. (a, b) Top view images of GDY-NS on the Cu substrate; (c) cross-sectional view of GDY-NS as an exfoliated sample; (d-f) TEM image of an exfoliated sample; (g) AFM image of GDY-NS on a Cu substrate; (h) schematic illustration of the structure of GDY-NS on a $\mathrm{Cu}$ substrate.

1.5406 Å. The X-ray photoelectron spectrometry (XPS) test was conducted using VG Scientific ESCA Lab220i-XL X-ray photoelectron spectrometer, using $\mathrm{Al} \mathrm{K} \alpha$ radiation as the excitation source. The Raman spectra were recorded at room temperature using a Thermo Scientific DXRXI system with excitation from an Ar laser at $532 \mathrm{~nm}$. In situ Raman spectroscopy was obtained using a three-electrode system. A GDY-NS film, separator, and sodium metal were assembled in the order from bottom to top, with drilling a hole through the middle of the separator and sodium metal to observe the GDY-NS electrode through a thin quartz glass. Nitrogen adsorption/desorption measurements were performed using a Quantachrome Autosorb gas-sorption system and calculated through the Brunauer-Emmett-Teller (BET) method. Galvanostatic charge/discharge tests were measured using a LAND-CT2001 instrument. Cyclic voltammetry (CV) and electrochemical impedance spectroscopy (EIS) were performed by using a CHI660 electrochemical workstation at each measurement condition.

\section{RESULTS AND DISCUSSION}

Structural and Morphology Analysis. As displayed in Figure 1, the morphology of the obtained GDY-NS was characterized by scanning electron microscopy (SEM). Figure $1 \mathrm{a}-\mathrm{c}$ shows the typical top and cross-sectional views of GDYNS, respectively. It is clearly observed that GDY-NS is arranged along the vertical direction on copper substrates and possessed large voids sub-micrometers in diameter that form a uniform 3D architecture structure on a larger scale. In Figure 1c, the thickness of GDY-NS is estimated around $400 \mathrm{~nm}$. The crosssectional images suggest that the 3D architecture and multiporous structure was present throughout the whole GDY-NS bulk, although the smooth surface of GDY-NS as an exfoliated sample could be observed closer to the copper side (Figure S1a,b in Supporting Information). The TEM image (Figure 1d,e) further confirms this 3D architecture structure at the nanoscale and demonstrates the porosity of GDY-NS. In Figure 1f, the interplanar distance of the layer of GDY-NS films is around $0.365 \mathrm{~nm}$ according to the HRTEM images, which is larger than that of graphite $(0.335 \mathrm{~nm})$, graphene $(0.350 \mathrm{~nm})$, and bulk GDY $(0.365 \mathrm{~nm}) .{ }^{29}$ Different from the crystal lattice, the high resolution TEM images displayed only lateral stacking without a cross structure, revealing that those streaks belong to the layered structure of GDY-NS. ${ }^{33}$ Figure $1 \mathrm{~g}$ shows AFM surface topographic images of GDY-NS. Results suggest that the obtained sample exhibited a uniform multiporous structure on a larger scale at $2.0 \times 2.0$ $\mu \mathrm{m}^{2}$. The value of the roughness parameter (root mean square) on GDY-NS films, obtained as $50.4 \mathrm{~nm}$, revealed a rough and porous surface morphology, which is associated with the large voids diameter of about $50 \mathrm{~nm}$ (Figure S2 in the Supporting Information). Meanwhile, the morphologies of GDY-NS with different reaction times and temperatures are shown to determine the formation of optimal GDY-NS, as suggested in Figure S3 (in Supporting Information). The schematic illustration of the GDY-NS is depicted in Figure $1 \mathrm{~h}$, similar as reported before. ${ }^{34}$

The structure characterizations of GDY-NS by XRD patterns, Raman, Fourier transform infrared (FT-IR) spectrum, XPS, and BET measurements are discussed in the Supporting Information. Briefly, in Figure 2a, the XRD patterns of the obtained samples show a broad peak at around $26.5^{\circ}$ similar to some highly disordered carbon materials, suggesting the poor 

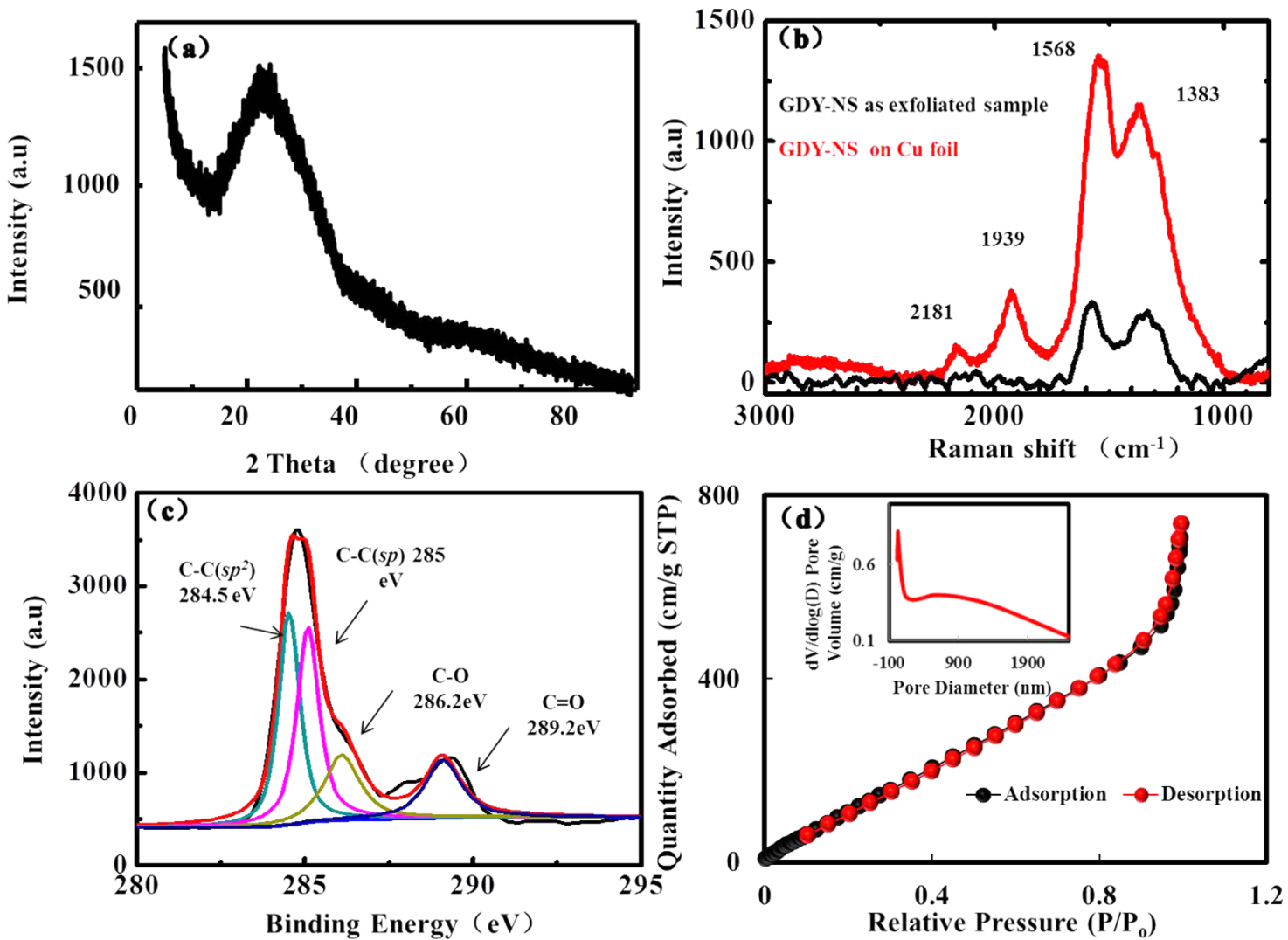

Figure 2. Structural characterization of GDY-NS. (a) XRD patterns of GDY-NS; (b) Raman spectra of GDY-NS on a Cu foil and as an exfoliated sample; (c) XPS survey of $\mathrm{C}^{1 \mathrm{~s}}$ binding energy profiles for GDY-NS; (d) $\mathrm{N}_{2}$ adsorption/desorption isotherms of GDY-NS (inset: pore-size distribution).

crystallinity of the sample, which may be due to random conformational fluctuation of the GDY-NS nanosheet on the mesoscopic scale. ${ }^{33}$ For the Raman spectrum shown in Figure $2 \mathrm{~b}$, the peak at $1383 \mathrm{~cm}^{-1}$ corresponds to the breathing vibration of $\mathrm{sp}^{2}$ carbon domains in aromatic rings ( $\mathrm{D}$ band) and the peak at $1568 \mathrm{~cm}^{-1}$ is due to the first-order scattering of the $\mathrm{E}_{2 \mathrm{~g}}$ mode for in-phase stretching vibration of the $\mathrm{sp}^{2}$ carbon lattice in aromatic rings ( $G$ band). A tiny peak at 1939 and $2181 \mathrm{~cm}^{-1}$ can be observed, which are ascribed to the butadiyne bond. ${ }^{35}$ XPS data shows that the synthetic products have both $\mathrm{sp}^{2}$ and $\mathrm{sp}$ hybrid carbon and the area ratio of the two are almost close to $1: 1$ (Figure 2c). ${ }^{36}$ The BET specific surface area of GDY-NS can reach to $467.9 \mathrm{~m}^{2} \mathrm{~g}^{-1}$, which is slightly lower than that previously reported for GDY as 873.76 $\mathrm{m}^{2} \mathrm{~g}^{-1}$. ${ }^{31}$ It is probably because GDY sheets stack randomly during the process of self-assembly and block a part of the micropore structure. The pore-size distribution analysis suggests a large number of mesopores and micropores exist from 1 to $50 \mathrm{~nm}$ according to the introduction of butadiyne linkages to form $18-\mathrm{C}$ hexagons and the benzene ring network. Meanwhile, abundant micropores (100-2000 nm) selfassembled from the GDY sheet were observed. Therefore, the mesopores, micropores, and high surface area were expected to facilitate sodium-ion transfer and increase the number of interfacial electrochemical sites, respectively. ${ }^{13,37}$

Na-Ion Battery Performance. The electrochemical performances of the binder-free GDY-NS electrodes are evaluated using half-cells with sodium metal as the counter electrode. As shown in Figure 3a, the cyclic voltammogram (CV) curves are summarized for the initial four cycles, with scan rate as $1 \mathrm{mV} \mathrm{s}^{-1}$. During the first cathodic scan, an irreversible band appears at around $0.8 \mathrm{~V}$, which is attributed to the formation of a solid electrolyte interface (SEI) film on the electrode surface similar to other carbon materials. ${ }^{6}$ It almost disappears from the second cycle, suggesting the formation of a stable SEI layer. As shown in Figure $3 b$, the charge-discharge curves of GDY-NS electrodes were in accordance with CV curves (Figure 3a) without an identified plateau during the reversible sodiation/desodiation process, which are similar to those of the reversible lithiation/lithiation process, as we reported previously. ${ }^{29-31}$ Figure $3 \mathrm{c}$ exhibited the initial chargedischarge capacities of $1492 / 812 \mathrm{mAh} \mathrm{g}^{-1}$ at the current density of $0.05 \mathrm{~A} \mathrm{~g}^{-1}$. It is noted that the initial coulombic efficiency is as high as $54.4 \%$ compared to that of other reports. ${ }^{13}$ The capacity loss of the initial charge-discharge capacities is probably attributed to the reductive decomposition of the electrolyte and the formation of a dense SEI film due to the amount of active sites and irreversible reaction between sodium ions and the butadiyne linkages and the residual functional group of the aromatic benzene rings. ${ }^{37}$ In Figure 3c, the rate capability of a GDY-NS film is evaluated at different current densities ranging from 0.05 to $5 \mathrm{~A} \mathrm{~g} \mathrm{~g}^{-1}$. The initial reversible capacities at various charge-discharge rates are retained at $812,760,697,527,402,278$, and $175 \mathrm{mAh} \mathrm{g}^{-1}$ at current densities of $0.05,0.1,0.2,0.5,1,2$, and $5 \mathrm{~A} \mathrm{~g}^{-1}$, respectively. When the current density is reset to $0.05 \mathrm{~A} \mathrm{~g}^{-1}$, the capacity increases to around $750 \mathrm{mAh} \mathrm{g}^{-1}$ and reveals the outstanding rate performance (Figure $3 \mathrm{c}$ ). On the basis of the cycles at a differential scan rate from 1 to $100 \mathrm{mV} \mathrm{s}^{-1}$, as summarized in Figure $3 \mathrm{~d}$, it could be observed that the 

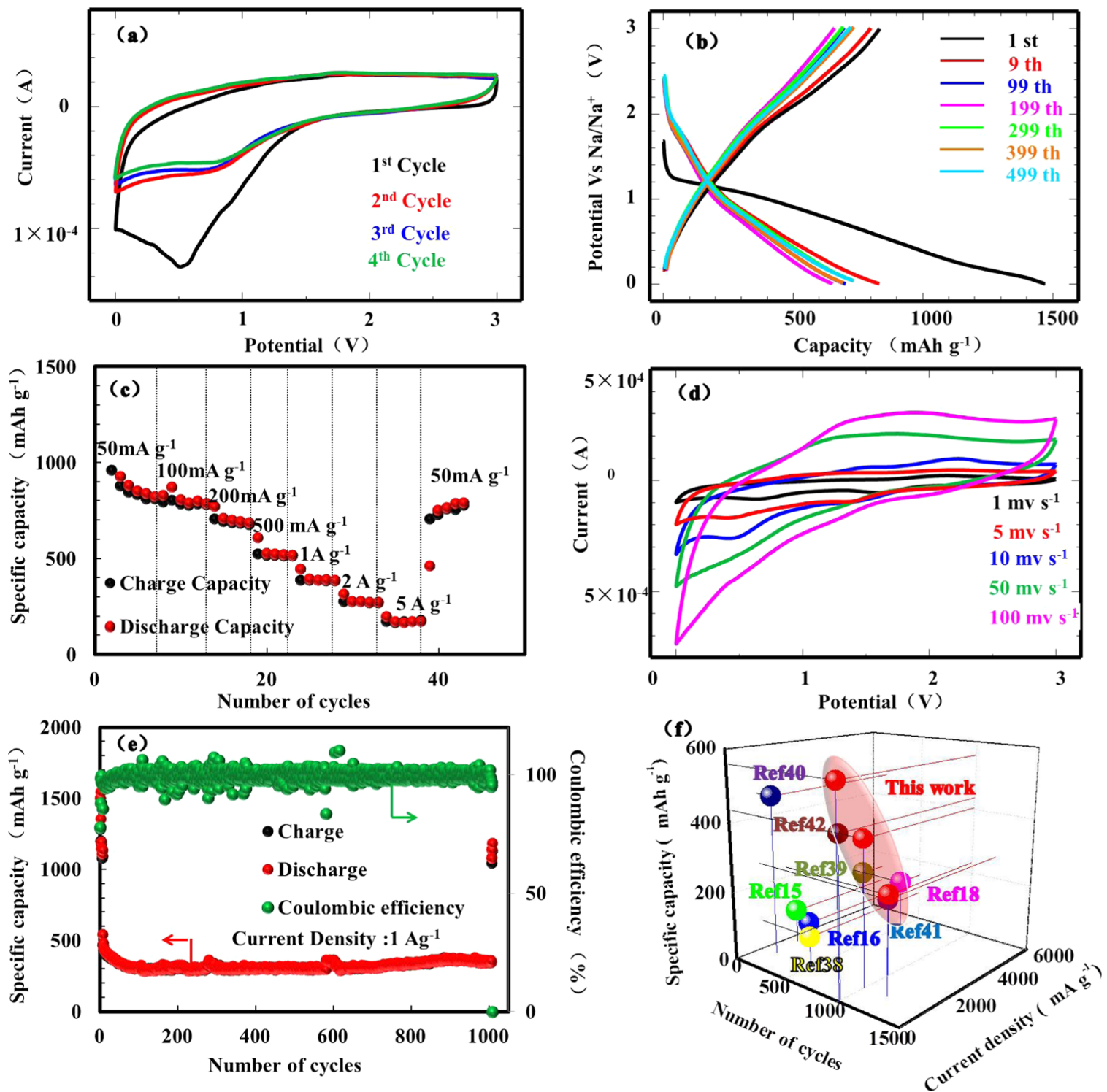

Figure 3. Electrochemical performance of GDY-NS electrodes in the sodium metal half-cell format; (a) CV profiles of GDY-NS electrodes for the initial four cycles, with the scan rate as $1 \mathrm{mV} \mathrm{s}^{-1}$; (b) the charge-discharge curves of GDY-NS electrodes at the current density of $50 \mathrm{~mA} \mathrm{~g}{ }^{-1}$, with different cycles; (c) the rate performance of the GDY-NS-based electrodes for NIBs, with current from $50 \mathrm{~mA} \mathrm{~g}^{-1}$ to $5 \mathrm{~A} \mathrm{~g}^{-1}$, respectively; (d) CV profiles of GDY-NS electrodes, with the scan rate from 1 to $100 \mathrm{mV} \mathrm{s}^{-1}$ at the first cycle, respectively; (e) the cycle performance of the productbased electrodes at the current density of $1 \mathrm{~A} \mathrm{~g}^{-1}$; (f) the NIB performance of GDY-NS and other references (all of the potential is vs $\mathrm{Na}^{+} /$ $\mathrm{Na}) .{ }^{15,16,18,38-42}$

potential peak is obviously moved from around 0.6 to $0.5 \mathrm{~V}$ when the scan rate increases from 1 to $100 \mathrm{mV} \mathrm{s}^{-1}$. In Figure $3 \mathrm{e}$, a reversible capacity of more than $405 \mathrm{mAh} \mathrm{g}^{-1}$ is shown after 1000 cycles at a current density of $1 \mathrm{~A} \mathrm{~g}^{-1}$, with a high coulombic efficiency of $>95 \%$, demonstrating the outstanding cycle performance. The initial reversible capacities decreased slightly at early cycles and maintained a reversible capacity of more than $562 \mathrm{mAh} \mathrm{g}^{-1}$ after 1000 cycles at a current density of $0.05 \mathrm{~A} \mathrm{~g} \mathrm{~g}^{-1}$ and $465 \mathrm{mAh} \mathrm{g}^{-1}$ after 1000 cycles at a current density of $0.5 \mathrm{~A} \mathrm{~g}^{-1}$ (Figure S7a,b). Therefore, the capacity retention ability, rate ability, and cycle ability of GDY-NS show a great advantage than those of other carbon nanomaterials, as summarized in Figure $3 \mathrm{f}^{13}$ The high specific capacity and long cycle life are attributed to the unique $3 \mathrm{D}$ architecture and multiporous structure with the butadiyne linkages that endow GDY-NS with abundant active sites for sodium storage and excellent chemical stability. ${ }^{33,37}$ The unique $3 \mathrm{D}$ architecture of
GDY-NS with numerous micropores and mesopores and the low barriers of sodium diffusion are helpful for rapid transport of sodium on the GDY-NS interface, thus making the GDY-NS suitable as sodium storage material even at high chargedischarge rates. It is noted that when the exfoliated GDY-NS powder acted as an anode electrode in NIBs with a high GDYNS mass loading around $1.02 \mathrm{mg} / \mathrm{cm}^{2}$, the capacity of GDY materials showed a significant decrease. The exfoliated GDYNS powder displays a steady and reversible capacity around 490 $\mathrm{mAh} \mathrm{g}^{-1}$ with current density $50 \mathrm{~mA} \mathrm{~g}^{-1}$, and less than 200 $\mathrm{mAh} \mathrm{g}^{-1}$ with current density $1 \mathrm{~A} \mathrm{~g}^{-1}$ (as shown in the Supporting Information in Figure $\mathrm{S} 8 \mathrm{~b}-\mathrm{d}$ ). This is probably caused by the stacking and interaction of the exfoliated GDYNS sheet, leading to the difficulty of sodium intercalation in bulk GDY than in thin and few-layered GDY-NS. Furthermore, the morphology of the GDY-NS electrode after 7 and 1000 cycles is shown in Figure S9a,b, respectively. It is directly 


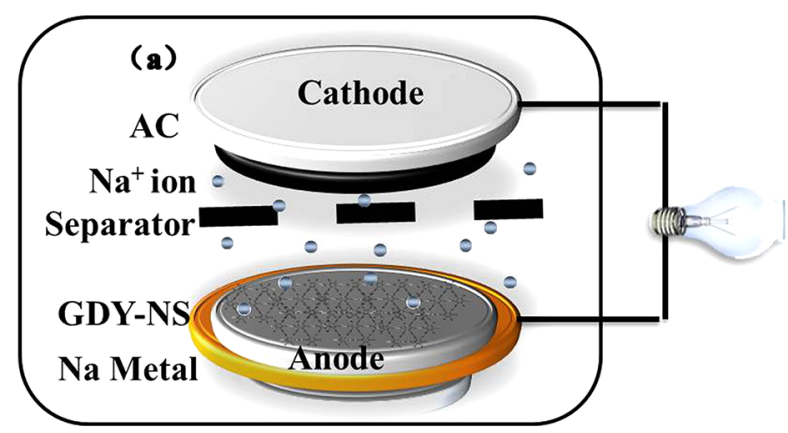

(b)
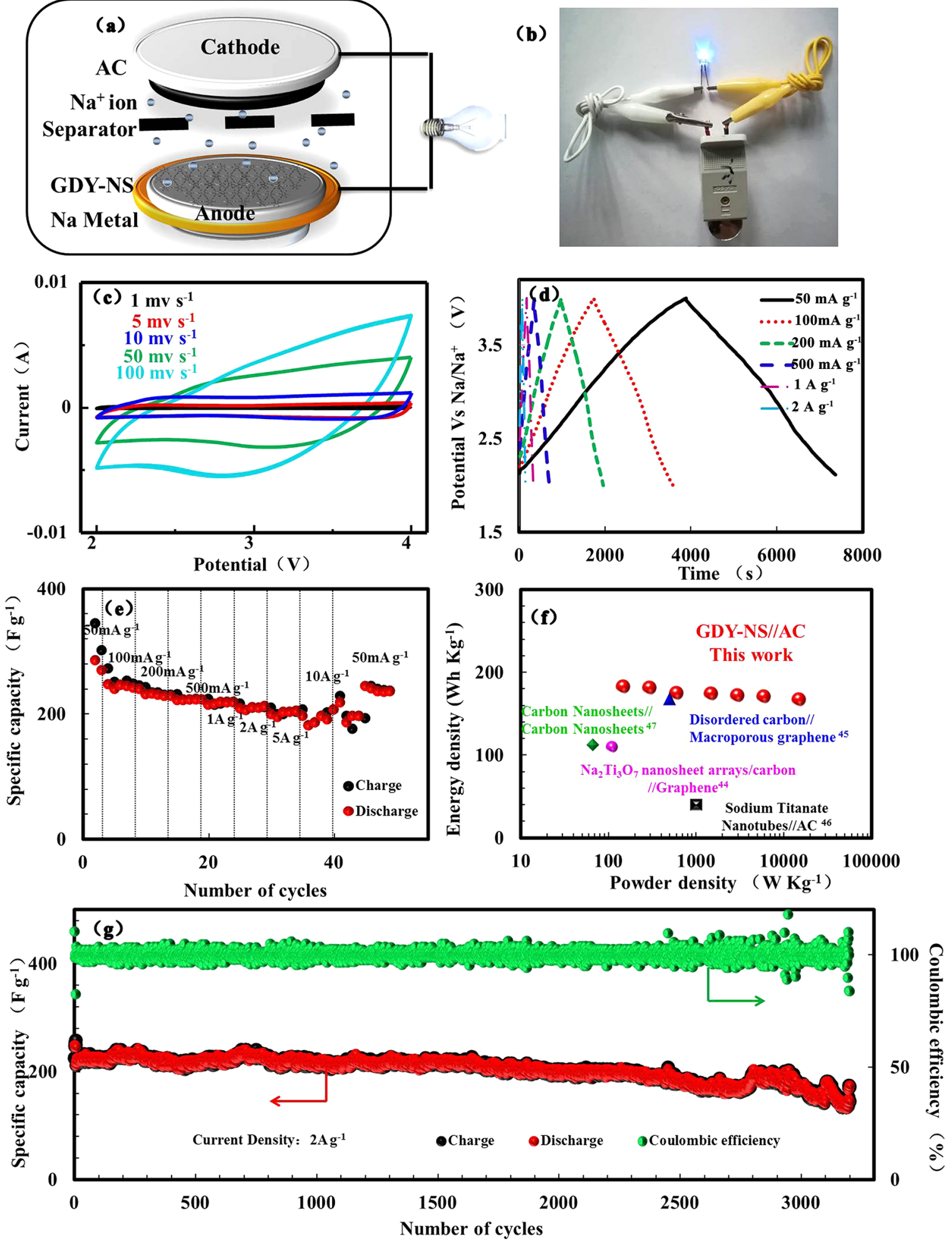

Figure 4. Electrochemical characterization of GDY-NS/AC NICs. (a) Schematic diagrams of the NIC full-cell configuration composed of the AC as the cathode and GDY-NS as the anode electrode; (b) lighting a blue LED by the GDY-NS/AC NIC cell; (c) CV profiles of GDY-NS electrodes with the scan rate from 1 to $100 \mathrm{mV} \mathrm{s}^{-1}$, respectively; (d, e) galvanostatic charge-discharge voltage profiles of GDY-NS electrodes at various current densities from $50 \mathrm{~mA} \mathrm{~g}^{-1}$ to $5 \mathrm{~A} \mathrm{~g} \mathrm{~g}^{-1}$, respectively; (f) Ragone plots of NICs with GDY-NS/AC and comparison of other references; ${ }^{42-47}$ and (g) the cycle performance of the GDY-NS/AC-applied NICs at the current density of $1 \mathrm{~A} \mathrm{~g}^{-1}$ (all of the potential is vs $\mathrm{Na}^{+} / \mathrm{Na}$ ).

observed that a thin SEI layer was formed on the surface of the GDY-NS film, resulting in low initial coulombic efficiency. Figure S9c,d shows the TEM image of GDY-NS for NIBs after 1000 cycles. According to the high resolution images, the interplanar distance of the GDY-NS layers is around $0.37 \mathrm{~nm}$ after 1000 cycles, indicating that sodium-ion intercalation into and deintercalation from the GDY layer would not change the layer structure of GDY greatly. Figure S9e (in the Supporting 
Information) showed Nyquist plots of GDY-NS electrodes before and after 1000 cycles. For details, the fitting kinetic parameters of the electrodes are listed in Table S1. It is noted that the Nyquist plots revealed that the diameter of the semicircle for the GDY-nanowire (NW) electrode before cycles in the high-medium frequency region was larger than that after cycling, along with the fitted $R_{\mathrm{ct}}$ value decreasing from 5.401 to $3.608 \Omega$ after 1000 cycles, leading to a stable cycle performance of the GDY-NW for LIB application. This phenomenon can be attributed to a stable SEI layer being formed after the initial cycle, which can effectively reduce the resistance of the interface and thus stabilize the battery system. ${ }^{31,38}$

Na-Ion Capacitor Performance. For the application of NICs, AC is chosen as the positive electrode material. Figure $4 \mathrm{a}, \mathrm{b}$ shows schematic diagrams of the NIC full-cell and operating a blue light-emitting diode (LED) through the cell, composed of the AC as the cathode and presodiation GDY-NS as the negative electrode consisting of extra sodium by direct attachment. For effective long term device operation, the optimal mass balance of the electrode is crucial. Because the specific reversible capacities of GDY-NS are around 812 and 46 $\mathrm{mAh} \mathrm{g}^{-1}$ for AC with a current density of $50 \mathrm{~mA} \mathrm{~g}^{-1}$ in the case of NIBs, respectively (Figure S10), the electrode mass ratio is optimized so as to balance the capacities for each electrode. Figure S11a shows the galvanostatic charge-discharge voltage profiles of GDY-NS electrodes at current densities of $0.05 \mathrm{~A} \mathrm{~g}^{-1}$ with different GDY-NS/AC mass ratios, respectively. In Figure S11b, the corresponding specific capacitances of the NICs incorporating GDY-NS as the negative electrode with different GDY-NS/AC mass ratios are exhibited. A better specific capacitance is exhibited as high as $237.6 \mathrm{~F} \mathrm{~g}^{-1}$ in accord with the smaller mass balance of the electrode. In Figure 4c, results suggest that at a high scan rate of $100 \mathrm{mV} \mathrm{s}^{-1}$, the CV curve has an early rectangular shape, indicating an ideal capacitive behavior with no obvious redox peaks, similar to that of GDY/AC LICs. ${ }^{31}$ It reveals that the AC cathode is the decisive one in the NIC devices even under existence of the sodium intercalation/deintercalation reaction for the GDY-NS anode. When the scan rate is as low as $1 \mathrm{mV} \mathrm{s}^{-1}$, the CV of the GDYNS electrode becomes a little distorted due to the behavior of the Faradaic reaction ( $\mathrm{Na}$ insertion/extraction reaction). ${ }^{39}$ Figure 4d,e suggests galvanostatic charge-discharge voltage profiles of GDY-NS electrodes at various current densities from 0.05 to $5 \mathrm{~A} \mathrm{~g}^{-1}$, respectively. The GDY-NS electrode possesses superior reversible specific capacitance with each current density. When the current density is increased from 0.05 to 5 $\mathrm{A} \mathrm{g}^{-1}$, the reversible specific capacitance shows a little decrease from 270 to $186 \mathrm{~F} \mathrm{~g}^{-1}$. When the current density is reset to $0.05 \mathrm{~A} \mathrm{~g}^{-1}$, the capacity is still as high as $216 \mathrm{~F} \mathrm{~g}^{-1}$, demonstrating an excellent rate performance. In Figure $4 \mathrm{f}$, the NIC delivers an initial specific energy as high as $182.3 \mathrm{Wh} \mathrm{kg}^{-1}$ at a power density of 300 and $166 \mathrm{Wh} \mathrm{kg}^{-1}$ at a power density of $15000 \mathrm{~W} \mathrm{~kg} \mathrm{~W}^{-1}$, presenting the benefit of better $\mathrm{Na}$-ion storage and delivery ability for GDY-NS than those of others reported. ${ }^{38,40,39,41}$ It is attributed to the high $3 \mathrm{D}$ porous structure that endows GDY-NS with abundant active sites for $\mathrm{Na}$ storage and excellent chemical stability of microporous and mesoporous GDY-NS. Figure $4 \mathrm{~g}$ exhibits the specific capacitance maintained as high as more than $200 \mathrm{~F} \mathrm{~g}^{-1}$ during the 3000 cycles at the current density of $1 \mathrm{~A} \mathrm{~g}^{-1}$, with high coulombic efficiency of $>90 \%$ from 5 th to 3000 th cycle. This demonstrates that the 3D architecture GDY-NS effectively improves sodium storage capacity and cycling ability and enhances diffusion kinetics. All of these results indicate that GDY-NS is a promising anode material both for NIBs and NICs with high capacity, outstanding rate capability, and a long cycle life.

Figure 5a shows the in situ Raman spectra series of the first charge/discharge process. The optical micrograph shows the
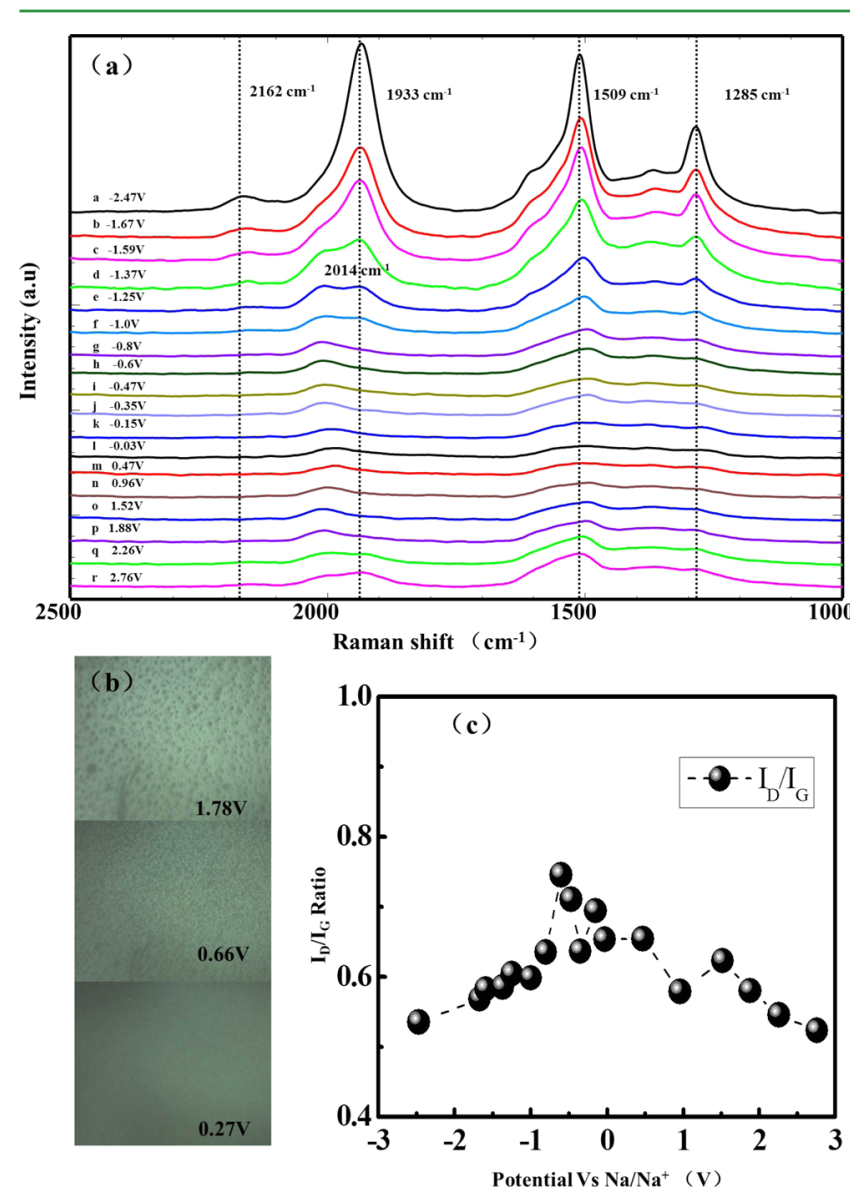

Figure 5. (a) In situ Raman spectra series of the first discharge process; (b) optical micrograph shows the accompanying changes in the color of the GDY-NS anode during the in situ sodiation at different voltages; (c) changing trend of the relative intensity between the $\mathrm{D}$ bond and $\mathrm{G}$ bond.

accompanying changes in the color of the GDY-NS anode during the in situ sodiation process, which was monitored at a discharge potential of $1.78,0.66$, and $0.27 \mathrm{~V}$, as shown in Figure $5 \mathrm{~b}$, respectively. There is an obvious color change when the discharge potential changes from 1.78 to $0.27 \mathrm{~V}$, suggesting the formation of SEI. In detail, the Raman results suggest that the butadiyne bond in $2162 \mathrm{~cm}^{-1}$ is gradually weakened and totally disappears under the SEI film formation potential (around 0.8 $\mathrm{V})$. It does not appear again even when the potential is increased during the process of charging, suggesting irreversible active butadiyne linkages act as sodium active sites through adsorption and deposition of electrolyte. Meanwhile, the intensity of stretching vibration of the conjugate butadiyne linkage at $1933 \mathrm{~cm}^{-1}$ is weakened and disappears gradually under the SEI film formation potential. In the process of charging, the peak of $1933 \mathrm{~cm}^{-1}$ reappears with gradually increased potential. However, a new peak at $2014 \mathrm{~cm}^{-1}$ is enhanced gradually above $0.35 \mathrm{~V}$ and shifted to the short wavelength under the potential for the sodium ions embedded 
in GDY butadiyne linkages (below $0.35 \mathrm{~V}$ ). Reversibly, the peaks of $2014 \mathrm{~cm}^{-1}$ show a redshift to a long wavelength with the potential increased from 0.03 to $2.76 \mathrm{~V}$. These results forcefully prove that the butadiyne linkages have contributed to the sodium-ion storage. ${ }^{32}$ Simultaneously, the intensity of the peak at 1285 and $1568 \mathrm{~cm}^{-1}$ are decreased during the first discharge process. For the $\mathrm{G}$ bond $\left(1568 \mathrm{~cm}^{-1}\right)$, it is moved to the short wavelength under the potential for the sodium ions embedded in the GDY sheet after the formation of the SEI film (below $0.8 \mathrm{~V}$ ) and shifted to long wavelength irreversibly with enhanced intensity during the charge process. Successively, the $I_{\mathrm{D}} / I_{\mathrm{G}}$ ratio increases with the increased potential, probably due to the added disordered carbon structure (shown in Figure 5c). At the SEI formation potential (about $0.8 \mathrm{~V}$ ), the $I_{\mathrm{D}} / I_{\mathrm{G}}$ ratio reaches the highest value. After the formation of the stable SEI film, the sodium ion is embedded and stored to form the GDY-Na intercalation compound. Therefore, the $I_{\mathrm{D}} / I_{\mathrm{G}}$ ratio reduces gradually as the discharge potential is reduced. In the process of charging, the $I_{\mathrm{D}} / I_{\mathrm{G}}$ ratio is undulated within a certain range because of the formation of stable SEI and part of the irreversible reaction of GDY-Na intercalation. ${ }^{37}$ Therefore, according to that previously reported, it is reasonable to consider that the specific capacity below $0.3 \mathrm{~V}$ corresponds to the origination from sodium intercalation into graphene-like interlayers. Meanwhile, the specific capacity from 0.3 to $0.8 \mathrm{~V}$ corresponds to the intercalation of sodium ion into GDY-NS films. The specific capacity above $0.8 \mathrm{~V}$ is associated with $\mathrm{Na}$ adsorption on the GDY-NS films or on the edge plane, similar to the formation of the graphite- $\mathrm{Li}$ intercalation compound. ${ }^{13}$

The proposed schematic of sodium-ion diffusion and the sodiation process in 3D architecture GDY-NS bulk, GDY-NS monolayers, and the GDY-NS molecular level for the NIB halfcell configuration are summarized in Figure 6. These results demonstrate the excellent cycle and rate performance of GDYNS-based device is benefitted from the controllable synthesis of the $3 \mathrm{D}$ architecture porous structure. The large electrolyte contact interface, short ion-diffusion path, and better accommodation of the insertion- and extraction-induced strain are attributed to the $3 \mathrm{D}$ architecture structure and rich butadiyne linkages. Therefore, the unique 3D architecture structure of GDY-NSs is able to improve the rapid transportation, the migration, and diffusion of sodium in the phase in GDY-NS from GDY monolayers to GDY sheets and the 3D architecture network, as suggested in Figure 6.

\section{CONCLUSIONS}

In summary, we reported the successful synthesis of $3 \mathrm{D}$ architecture GDY-NS with porous structure and its application for high-performance sodium storage devices included NIBs and NICs. Results proved that the existence of butadiyne linkages played an important role in increasing the sodium bonding site and promote the formation of a stable SEI layer, which was confirmed through the in situ Raman measurement. The contribution of the $3 \mathrm{D}$ architecture structure and the butadiyne linkages of the GDY-NS substantially optimize sodium storage behavior through the enhanced activity sites, accommodating a large volume porous structure and facilitating a fast ion-diffusion channel. The GDY-NS exhibits a stable reversible capacity of approximately $400 \mathrm{mAh} \mathrm{g}^{-1}$ over 1000 cycles at a current density of $1 \mathrm{~A} \mathrm{~g}^{-1}$ in half-cells for NIBs, with an excellent rate and long cycle performance. Thus GDY-NS films could deliver a capacitance more than $200 \mathrm{~F} \mathrm{~g}^{-1}$ over 3000 cycles at $1 \mathrm{~A} \mathrm{~g}^{-1}$ for NICs with an active carbon cathode and

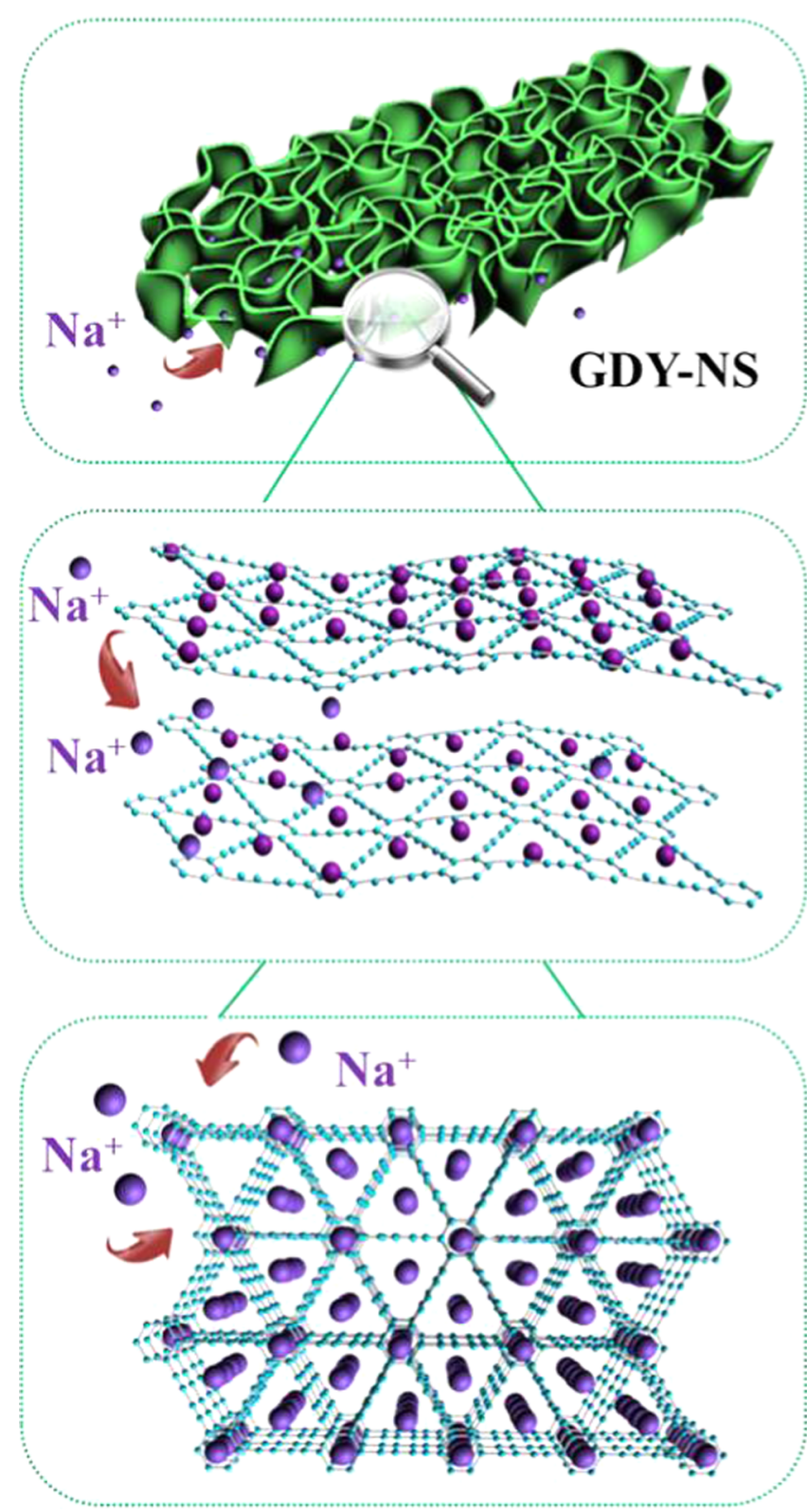

Figure 6. Proposed schematic diagrams of the sodium-ion diffusion and sodiation in 3D architecture GDY-NS materials, GDY-NS monolayers, and GDY-NS molecular structure for the NIB half-cell configuration, respectively.

exhibit an initial specific energy as high as $182.3 \mathrm{Wh} \mathrm{kg}^{-1}$ at a power density of $300 \mathrm{~W} \mathrm{~kg}^{-1}$. Results indicate that the obtained GDY-NS films showed interesting properties and could be a potential competitor for application in large-scale sodium rechargeable batteries.

\section{ASSOCIATED CONTENT}

\section{S Supporting Information}

The Supporting Information is available free of charge on the ACS Publications website at DOI: 10.1021/acsami.7b11420.

Additional analysis of FT-IR, UV-vis, XPS, SEM, AFM, CVs, EIS for GDY-NS (PDF) 


\section{AUTHOR INFORMATION}

\section{Corresponding Author}

*E-mail: huangcs@qibebt.ac.cn.

\section{ORCID}

Changshui Huang: 0000-0001-5169-0855

\section{Author Contributions}

${ }^{\S}$ K.W. and N.W. contributed equally to this work.

\section{Notes}

The authors declare no competing financial interest.

\section{ACKNOWLEDGMENTS}

This study was supported by the National Natural Science Foundation of China (21790050, 21790051, and 21771187), the Hundred Talents Program and Frontier Science Research Project (QYZDB-SSW-JSC052) of the Chinese Academy of Sciences, and the Natural Science Foundation of Shandong Province (China) for Distinguished Young Scholars (JQ201610).

\section{REFERENCES}

(1) Armand, M.; Tarascon, J. M. Building Better Batteries. Nature 2008, 451, 652-657.

(2) Braga, M. H.; Grundish, N. S.; Murchison, A. J.; Goodenough, J. B. Alternative Strategy for A Safe Rechargeable Battery. Energy Environ. Sci. 2017, 10, 331-336.

(3) Lin, D.; Liu, Y.; Cui, Y. Reviving the Lithium Metal Anode for High-Energy Batteries. Nat. Nanotechnol. 2017, 12, 194-206.

(4) Zheng, Y. H.; Wang, Y. S.; Lu, Y. X.; Hu, Y. S.; Li, J. A HighPerformance Sodium-Ion Battery Enhanced by Macadamia Shell Derived Hard Carbon Anode. Nano Energy 2017, 489-498.

(5) Kim, S. W.; Seo, D. H.; Ma, X. H.; Ceder, G.; Kang, K. Electrode Materials for Rechargeable Sodium-Ion Batteries: Potential Alternatives to Current Lithium-Ion Batteries. Adv. Energy Mater. 2012, 2, $710-712$.

(6) Yabuuchi, N.; Kubota, K.; Dahbi, M.; Komaba, S. Research Development on Sodium-Ion Batteries. Chem. Rev. 2014, 114, 1163611682 .

(7) Thackeray, M. M.; Johnson, P. J.; de Picciotto, L. A.; Bruce, P. G.; Goodenough, J. B. Electrochemical Extraction of Lithium from $\mathrm{LiMn}_{2} \mathrm{O}_{4}$. Mater. Res. Bull. 1984, 19, 179-187.

(8) Ellis, B. L.; Makahnouk, W. R. M.; Makimura, Y.; Toghill, K.; Nazar, L. F. A Multifunctional 3.5 V Iron-Based Phosphate Cathode for Rechargeable Batteries. Nat. Mater. 2007, 6, 749-753.

(9) Delmas, C.; Cherkaoui, F.; Nadiri, A.; Hagenmuller, P. A. Nasicon-Type Phase as Intercalation Electrode: $\mathrm{NaTi}_{2}\left(\mathrm{PO}_{4}\right)_{3}$. Mater. Res. Bull. 1987, 22, 631-639.

(10) Wu, X. Y.; Ma, J.; Ma, Q. D.; Xu, S. Y.; Hu, Y. S.; Sun, Y.; Li, H.; Chen, L. Q.; Huang, X. J. A Spray Drying Approach for The Synthesis of A $\mathrm{Na}_{2} \mathrm{C}_{6} \mathrm{H}_{2} \mathrm{O}_{4} / \mathrm{CNT}$ Nanocomposite Anode for Sodium-Ion Batteries. J. Mater. Chem. A 2015, 3, 13193-13197.

(11) Chen, Z.; Belharouak, I.; Sun, Y. K.; Amine, K. Titanium-Based Anode Materials for Safe Lithium-Ion Batteries. Adv. Funct. Mater. 2013, 23, 959-969.

(12) Luo, W.; Shen, F.; Bommier, C.; Zhu, H.; Ji, X. L.; Hu, L. B. NaIon Battery Anodes: Materials and Electrochemistry. Acc. Chem. Res 2016, 49, 231-240.

(13) Hou, H. S.; Qiu, X. Q.; Wei, W. F.; Zhang, Y.; Ji, X. B. Carbon Anode Materials for Advanced Sodium-Ion Batteries. Adv. Energy Mater. 2017, No. 1602898.

(14) Zhu, C.; Mu, X.; van Aken, P. A.; Yu, Y.; Maier, J. SingleLayered Ultrasmall Nanoplates of $\mathrm{MoS}_{2}$ Embedded in Carbon Nanofibers with Excellent Electrochemical Performance for Lithium and Sodium Storage. Angew. Chem., Int. Ed. 2014, 53, 2152-2156.

(15) Tang, K.; Fu, L.; White, R. J.; Yu, L.; Titirici, M.; Antonietti, M.; Maier, J. Hollow Carbon Nanospheres with Superior Rate Capability for Sodium-Based Batteries. Adv. Energy Mater. 2012, 2, 873-877.
(16) Xiao, L.; Cao, Y.; Henderson, W. A.; Sushko, M. L.; Shao, Y.; Xiao, J.; Wang, W.; Engelhard, M. H.; Nie, Z.; Liu, J. Hard Carbon Nanoparticles as High-Capacity, High-Stability Anodic Materials for Na-Ion Batteries. Nano Energy 2016, 19, 279-288.

(17) Cohn, A. P.; Share, K.; Carter, R.; Oakes, L.; Pint, C. L. Ultrafast Solvent-Assisted Sodium Ion Intercalation into Highly Crystalline Few-Layered Graphene. Nano Lett. 2016, 16, 543-548.

(18) Deng, X.; Xie, K.; Li, L.; Zhou, W.; Sunarso, J.; Shao, Z. Scalable Synthesis of Self-Standing Sulfur-Doped Flexible Graphene Films as Recyclable Anode Materials for Low-Cost Sodium-Ion Batteries. Carbon 2016, 107, 67-73.

(19) Palomares, V.; Serras, P.; Villaluenga, I.; Hueso, K. B.; Carretero-Gonzalez, J.; Rojo, T. Na-Ion Batteries, Recent Advances and Present Challenges to Become Low Cost Energy Storage Systems. Energy Environ. Sci. 2012, 5, 5884-5901.

(20) Wang, X.; Kajiyama, S.; linuma, H.; Hosono, E.; Oro, S.; Moriguchi, I.; Okubo, M.; Yamada, A. Pseudocapacitance of MXene Nanosheets for High-Power Sodium-Ion Hybrid Capacitors. Nat. Commun. 2015, 6, No. 6544.

(21) Stoller, M. D.; Park, S.; Zhu, Y.; An, J.; Ruoff, R. S. GrapheneBased Ultracapacitors. Nano Lett. 2008, 8, 3498-3502.

(22) Kuratani, K.; Yao, M.; Senoh, H.; Takeichi, N.; Sakai, T.; Kiyobayashi, T. Na-Ion Capacitor Using Sodium Pre-doped Hard Carbon and Activated Carbon. Electrochim. Acta 2012, 76, 320-325.

(23) Chen, Z.; Augustyn, V.; Jia, X.; Xiao, Q.; Dunn, B.; Lu, Y. HighPerformance Sodium-Ion Pseudocapacitors Based on Hierarchically Porous Nanowire Composites. ACS Nano 2012, 6, 4319-4327.

(24) Li, Y.; Xu, L.; Liu, H.; Li, Y. Graphdiyne and graphyne: from theoretical predictions to practical construction. Chem. Soc. Rev. 2014, 43, 2572-2586.

(25) Parvin, N.; Jin, Q.; Wei, Y.; Yu, R.; Zheng, B.; Huang, L.; Zhang, Y.; Wang, L. H.; Zhang, H.; Gao, M. Y.; Zhao, H. J.; Hu, W. P.; Li, Y. L.; Wang, D. Few-Layer Graphdiyne Nanosheets Applied for Multiplexed Real-Time DNA Detection. Adv. Mater. 2017, 29, No. 1606755.

(26) Xu, J.; Li, J. Q.; Yang, Q. L.; Xiong, Y.; Chen, C. G. In-Situ Synthesis of $\mathrm{MnO}_{2} @$ Graphdiyne Oxides Nanocomposite With Enhanced Performance of Supercapacitors. Electrochim. Acta 2017, $251,672-680$.

(27) Srinivasu, K.; Ghosh, S. K. Graphyne and Graphdiyne: Promising Materials for Nanoelectronics and Energy Storage Applications. J. Phys. Chem. C 2012, 116, 5951-5956.

(28) Li, G. X.; Li, Y. L.; Liu, H. B.; Guo, Y. B.; Li, Y. J.; Zhu, D. B. Architecture of Graphdiyne Nanoscale Films. Chem. Commun. 2010, 46, 3256-3258

(29) Huang, C. H.; Zhang, S. L.; Liu, H. B.; Yong, Y. J.; Cui, G. L.; Li, Y. L. Graphdiyne for High Capacity and Long-Life Lithium Storage. Nano Energy 2015, 11, 481-489.

(30) Zhang, S.; Du, H.; He, J.; Huang, C.; Liu, H.; Cui, G.; Li, Y. Nitrogen-Doped Graphdiyne Applied for Lithium-Ion Storage. ACS Appl. Mater. Interfaces 2016, 8, 8467-8473.

(31) Du, H. P.; Yang, H.; Huang, C. S.; He, J. J.; Liu, H. B.; Li, Y. L. Graphdiyne Applied for Lithium-Ion Capacitors Displaying High Power and Energy Densities. Nano Energy 2016, 22, 615-622.

(32) Xu, Z. M.; Lv, X. J.; Li, J.; Chen, J. G.; Liu, Q. S. A Promising Anode Material for Sodium-Ion Battery With High Capacity and High Diffusion Ability: Graphyne and Graphdiyne. RSC Adv. 2016, 6, 25594-25600.

(33) Zhang, S. L.; He, J. J.; Zheng, J.; Huang, C. S.; Lv, Q.; Wang, K.; Wang, N.; Lan, Z. G. Porous Graphdiyne Applied for Sodium Ion Storage. J. Mater. Chem. A 2017, 5, 2045-2051.

(34) Zhou, J.; Gao, X.; Liu, R.; Xie, Z.; Yang, J.; Zhang, S.; Zhang, G.; Liu, H.; Li, Y.; Zhang, J.; Liu, Z. F. Synthesis of Graphdiyne Nanowalls Using Acetylenic Coupling Reaction. J. Am. Chem. Soc. 2015, 137, 7596-7599.

(35) Gao, X.; Zhou, J.; Du, R.; Xie, Z.; Deng, S.; Liu, R.; Liu, Z.; Zhang, J. Robust Superhydrophobic Foam: A Graphdiyne-Based Hierarchical Architecture for Oil/Water Separation. Adv. Mater. 2016, $28,168-173$. 
(36) Li, J.; Gao, X.; Liu, B.; Feng, Q.; Li, X. B.; Huang, M. Y.; Liu, Z.; Zhang, J.; Tung, C. H.; Wu, L. Z. Graphdiyne: A Metal-Free Material as Hole Transfer Layer To Fabricate Quantum Dot-Sensitized Photocathodes for Hydrogen Production. J. Am. Chem. Soc. 2016, 138, 3954-3957.

(37) Niaei, A. H. F.; Hussain, T.; Hankel, M.; Searles, D. J. SodiumIntercalated Bulk Graphdiyne as An Anode Material for Rechargeable Batteries. J. Power Sources 2017, 343, 354-363.

(38) Yan, Y.; Yin, Y.-X.; Guo, Y.-G.; Wan, L.-J. A Sandwich-Like Hierarchically Porous Carbon/Graphene Composite as A HighPerformance Anode Material for Sodium-Ion Batteries. Adv. Energy Mater. 2014, 8, No. 1301584.

(39) Wang, F.; Wang, X.; Chang, Z.; Wu, X.; Liu, X.; Fu, L.; Zhu, Y.; $\mathrm{Wu}$, Y.; Huang, W. A Quasi-Solid-State Sodium-Ion Capacitor with High Energy Density. Adv. Mater. 2015, 27, 6962-6968.

(40) Dong, S. Y.; Shen, L. F.; Li, H. S.; Pang, G.; Dou, H.; Zhang, X. G. Flexible Sodium-Ion Pseudocapacitors Based on 3D $\mathrm{Na}_{2} \mathrm{Ti}_{3} \mathrm{O}_{7}$ Nanosheet Arrays/Carbon Textiles Anodes. Adv. Funct. Mater 2016, 26, 3703-3710.

(41) Yin, J.; Qi, L.; Wang, H. Y. Sodium Titanate Nanotubes as Negative Electrode Materials for Sodium-Ion Capacitors. ACS Appl. Mater. Interfaces 2012, 4, 2762-2768.

(42) Yang, J.; Zhou, X.; Wu, D.; Zhao, X.; Zhou, Z. S-Doped N-Rich Carbon Nanosheets with Expanded Interlayer Distance as Anode Materials for Sodium-Ion Batteries. Adv. Mater. 2017, No. 1604108.

(43) Hou, H.; Shao, L.; Zhang, Y.; Zou, G.; Chen, J.; Ji, X. Large-Area Carbon Nanosheets Doped with Phosphorus: A High-Performance Anode Material for Sodium-Ion Batteries. Adv. Sci. 2017, 4, No. 1600243.

(44) Xu, J.; Wang, M.; Wickramaratne, N. P.; Jaroniec, M.; Dou, S.; Dai, L. High-Performance Sodium Ion Batteries Based on a 3D Anode from Nitrogen-Doped Graphene Foams. Adv. Mater. 2015, 27, 20422048.

(45) Fan, L.; Lu, B. Reactive Oxygen-Doped 3D Interdigital Carbonaceous Materials for $\mathrm{Li}$ and $\mathrm{Na}$ Ion Batteries. Small 2016, 12, 2783-2791.

(46) Sun, T.; Li, X.; Wang, H.; Bao, D.; Meng, F.; Zhang, X. A Biodegradable Polydopamine-Derived Electrode Material for HighCapacity and Long-Life Lithium-Ion and Sodium-Ion Batteries. Angew. Chem., Int. Ed. 2016, 55, 10662-10666.

(47) Wang, H. L.; Mitlin, D.; Ding, J.; Li, Z.; Cui, K. Excellent Energy-Power Characteristics from A Hybrid Sodium Ion Capacitor Based on Identical Carbon Nanosheets in Both Electrodes. J. Mater. Chem. A 2016, 4, 5149-5158. 